\title{
Running of the Spectral Index and Violation of the Consistency Relation Between Tensor and Scalar Spectra from trans-Planckian Physics
}

\author{
A. Ashoorioon ${ }^{1}$, J. L. Hovdebo ${ }^{2}$, R. B. Mann $^{3}$ \\ Department of Physics, University of Waterloo, \\ Waterloo, Ontario, N2L 3G1, Canada
}

\begin{abstract}
One of the firm predictions of inflationary cosmology is the consistency relation between scalar and tensor spectra. It has been argued that such a relation -if experimentally confirmed - would offer strong support for the idea of inflation. We examine the possibility that trans-Planckian physics violates the consistency relation in the framework of inflation with a cut-off proposed in astro-ph/0009209. We find that despite the ambiguity that exists in choosing the action, Planck scale physics modifies the consistency relation considerably. It also leads to the running of the spectral index. For modes that are larger than our current horizon, the tensor spectral index is positive. For a window of $k$ values with amplitudes of the same order of the modes which are the precursor to structure formation, the behavior of tensor spectral index is oscillatory about the standard Quantum field theory result, taking both positive and negative values. There is a hope that in the light of future experiments, one can verify this scenario of short distance physics.
\end{abstract}

\footnotetext{
1amjad@astro.uwaterloo.ca

2jlhovdeb@sciborg.uwaterloo.ca

${ }^{3}$ mann@avatar.uwaterloo.ca
} 


\section{Introduction}

One of the intriguing properties of inflationary cosmology, which could be used to test the fundamental theories of quantum gravity, is its capacity to accommodate sub-Planckian fluctuations that were redshifted exponentially during a quasi-de-Sitter expansion of the universe [1]. Many realizations of inflation predict several more e-foldings than are required to solve the problems of standard cosmology [2]. Assuming that these inflationary models are correct, all scales of cosmological interest today originate inside the Planck scale at the early stages of inflation. These fluctuations would be manifest in the temperature anisotropy of the cosmic microwave background radiation (CMBR), which can be regarded as a fossil record of primeval inhomogeneities. It is therefore reasonable to expect that by studying the cosmic microwave background radiation one can extract information about physics at very small distance scales [4].

Several approaches have been employed to find out how modifications of Planck scale physics affect orthodox calculations of the power spectrum in the context of Quantum Field Theory. Originally, Brandenberger and Martin considered the effect of Planck scale physics to be encoded in modifications to dispersion relations [3]. Similar methods were employed to investigate the same phenomenon in the context of black hole physics $[5,6]$. These modifications were inspired by higher dimensional models of the universe [7] or from condensed matter analogs of gravity [8]. It was shown that the prediction of a thermal Hawking spectrum is insensitive to modifications of the physics at the trans-Planckian end of the spectrum.

In an inflationary setting Planck scale physics may or may not leave an imprint, depending on whether the mode behaves adiabatically when its wavelength is smaller than the cut-off scale. Nonadaibatic evolution of a mode when its wavelength is smaller than the Planck scale results in an excited state at the time that the wavelength crosses the Hubble radius during inflation [9]. However, since this leads to a large amount of particle production by trans-Planckian physics, it has been claimed that such a possibility is excluded $[10,11]$.

Danielsson proposed a method in which our lack of knowledge about Planck scale physics is parametrized in the choice of state at the time a mode reaches the minimum scale [12]. The state at the minimum scale-crossing is the state of minimized uncertainty. Modifications to the standard analysis are of order $H / \Lambda$, where $H$ is the Hubble parameter during inflation

and $\Lambda$ is the scale at which new physics appears. This scenario was later generalized to power-law backgrounds in [13].

A beautiful mechanism was suggested in [14] to incorporate minimum length into the 
inflationary formalism. The only assumption underlying this formalism is that the fundamental theory of quantum gravity possesses a linear operator $X^{i}$ for every space-time coordinate and that its expectation value $\left\langle X^{i}\right\rangle$ is real. One can then show that the short distance structure of any such coordinate could not only be continuous or discrete, but could also be unsharp in one of two ways [15]. The two unsharp cases are distinguished by the so-called deficiency indices of $X^{i}$ being either nonzero and equal (Fuzzy type A) or unequal (Fuzzy type B) [15]. In the case of fuzzy type B, sequences of vectors in the physical domain exist such that $\triangle X^{i}$ converges to zero. They are fuzzy in the sense that vectors of increasing localization around different expectation values in general do not become orthogonal. Fuzzy type A behavior has appeared in a number of studies in quantum gravity and string theory where the uncertainty in $\triangle X^{i}$ has a finite lower bound at Planck scale [16]. This short distance structure can be modelled as quantum gravitational correction to the commutation relation between the position and momentum operators

$$
[\mathbf{X}, \mathbf{P}]=i\left(1+\beta \mathbf{P}^{2}\right)
$$

where $\beta^{1 / 2}$ parameterizes the minimum length. The equations for tensor modes were later analyzed numerically in $[17,18]$, and it was predicted that the effect on the CMBR can be as large as $\sigma$, where $\sigma$ is the ratio of the minimum length to the Hubble length during inflation, $\beta^{1 / 2} H \equiv \sigma$.

Quite recently it was discovered in [19] that this mechanism of implementing minimal length in the action has an ambiguity: The usual strategy for determining the initial condition requires reformulating the action and discarding a boundary term. In the absence of minimal length, two actions that differ by a boundary term are equivalent. However, the introduction of a minimal length scale renders two actions that normally differ by a boundary term inequivalent, yielding different equations of motion. One has an infinite set of actions that are equivalent when the minimal length is set to zero. Only experiment can adjudicate which choice of action is preferable. Nevertheless, in [20], from the infinite number of actions that are equivalent in the absence of minimal length, we adopt two actions for each of tensor and scalar fluctuations. The first one is chosen by a minimalist criterion: we select the action that is derived directly by expanding the action of a scalar field minimally coupled to gravity without introducing any additional terms by hand. The second action, which differs from the first by a boundary term, is chosen by the criterion of similarity with the action of a free massive scalar field in a Minkowskian background. Such a similarity simplifies the task of choosing the vacuum. Basically, one can choose the vacuum as one does in Minkowskian 
space-time.

A simple, near-de-Sitter background has recently been investigated in this context [20], where it was shown that the tensor/scalar ratio, gets modified as one incorporates minimal length. Trans-Planckian physics may or may not leave its thumbprint on this ratio depending on the actions one chooses for tensor and scalar perturbations. Such an ambiguity was also employed in [21] to account for the existence of cosmic magnetic fields. In this article, we consider the implications of minimal length in a power-law background to find the possible scale-dependence of the tensor/scalar ratio.

We will examine, in the context of the minimal length hypothesis as implemented in [14], a firm prediction of inflationary cosmology, the consistency relation between scalar and tensor perturbations. In the case of single-field slow-roll inflation the consistency conditions are given in terms of equality relations, whereas for multiple-field models of inflation these are weakened to inequalities. The first of the consistency relations states that the ratio of the amplitude of tensor to scalar perturbations is a constant known as the tensor spectral index [22]. We investigate how the effects of trans-Planckian physics alter this ratio and the tensor spectral index under the considerations noted in [14]. This is in contrast to recent work in this area in which this possibility was investigated without focusing on any specific model of short distance physics, instead assuming that the trans-Planckian energies result in a vacuum state that is different from the standard Bunch-Davies vacuum [23]. We shall restrict ourselves to single-field inflation for the rest of the discussion, although our results could straightforwardly be generalized to multiple-field inflation.

Our paper is structured as follows: first we recapitulate our results [19] for both tensor and scalar fluctuations. Next, we study numerically the equations of motion for scalar and tensor modes in a power-law background and derive the tensor/scalar fluctuations and the tensor spectral index in each case. As mentioned earlier, actions that differ by a boundary term are rendered inequivalent once one implements the minimal length hypothesis. Although this implies an infinite amount of freedom in choosing the action for both scalar and tensor fluctuations, there are only a few actions that have reasonable physical motivation, and we shall confine our considerations to these cases. Specifically, we shall discuss how these physically well-motivated but distinct actions modify the consistency relation between tensor and scalar spectra. 


\section{Scalar and Tensor Perturbations with Minimum Length}

To find the action for scalar and tensor perturbations, we expand the action of a scalar field minimally coupled to gravity

$$
S=\frac{1}{2} \int\left(\partial_{\mu} \phi \partial^{\mu} \phi-V(\phi)\right) \sqrt{-g} d^{4} x-\frac{1}{16 \pi G} \int R \sqrt{-g} d^{4} x
$$

using the most general form of the metric with scalar and tensor fluctuations

$$
d s^{2}=a^{2}(\tau)\left[(1+2 \Phi) d \tau^{2}-\left((1-2 \Psi) \delta_{i j}+h_{i j}\right) d y^{i} d y^{j}\right]
$$

where $\Phi$ and $\Psi$ are scalar fields and $h_{i j}$ is a symmetric tensor field satisfying transverse traceless gauge, $h_{i}^{i}=h_{i j}{ }^{j}=0$. At the same time, we also perturb the inflaton field about its homogeneous background value

$$
\phi(\tau)=\phi_{0}(\tau)+\delta \phi
$$

Here $\phi_{0}$ is the homogeneous part that drives inflation and $\delta \phi \ll \phi_{0}$. Also $\tau$ is the conformal time and $a(\tau)$ the scale factor of the inflating spatially flat background.

Using Eqs.(3) and (4) to expand action (2) to second order, the action for scalar perturbations can be written in terms of the intrinsic curvature perturbations of the comoving hypersurface, $\Re=-\frac{a^{\prime}}{a} \frac{\delta \phi}{\phi_{0}^{\prime}}-\Psi$, in the following form [19]

$$
S_{S}^{(1)}=\frac{1}{2} \int d \tau d^{3} \mathbf{y} z^{2}\left(\left(\partial_{\tau} \Re\right)^{2}-\delta^{i j} \partial_{i} \Re \partial_{j} \Re\right),
$$

where $\partial_{i}$ denotes differentiation with respect to spatial coordinates and

$$
z=\frac{a \phi_{0}^{\prime}}{\alpha}, \quad \alpha=a^{\prime} / a
$$

The prime denotes differentiation with respect to $\tau$. The quantity $\Re$ is a gauge-invariant combination of scalar fluctuations of the metric and inflaton perturbations [24].

Incorporating the minimal length hypothesis involves retaining the action (2) as above, but modifying the underlying position-momentum commutation relation similar to eq.(1). Specifically, the first order form of the commutation relation in $\beta$ has the form [14]:

$$
\left[\mathbf{X}^{i}, \mathbf{P}^{j}\right]=i\left(\frac{2 \beta p^{2}}{\sqrt{1+4 \beta p^{2}}-1} \delta^{i j}+2 \beta \mathbf{P}^{i} \mathbf{P}^{j}\right)
$$

whose Hilbert space representation can be conveniently written as

$$
\mathbf{X}^{i} \psi(\rho)=i \partial_{\rho^{i}} \psi(\rho) \quad \mathbf{P}^{i} \psi(\rho)=\frac{\rho^{i}}{1-\beta \rho^{2}} \psi(\rho)
$$


where the scalar product of two quantum fields is

$$
\left(\psi_{1}, \psi_{2}\right)=\int_{\rho^{2}<\beta^{-1}} d^{3} \rho \psi_{1}^{*}(\rho) \psi_{2}(\rho)
$$

It is then straightforward to derive the cutoff modified equation of motion for the fluctuation mode $u_{\tilde{k}}$, which is [19]

$$
u_{\tilde{k}}^{\prime \prime}+\frac{\kappa^{\prime}}{\kappa} u_{\tilde{k}}^{\prime}+\left(\mu-\frac{z^{\prime \prime}}{z}-\frac{z^{\prime} \kappa^{\prime}}{z \kappa}\right) u_{\tilde{k}}=0
$$

where $u_{\tilde{k}} \equiv z \Re_{\tilde{k}}$ and

$$
\begin{aligned}
& \mu(\tau, \tilde{k})=-\frac{a^{2}}{\beta} \frac{W\left(-\beta \tilde{k}^{2} / a^{2}\right)}{\left(1+W\left(-\beta \tilde{k}^{2} / a^{2}\right)\right)^{2}} \\
& \kappa(\tau, \tilde{k})=\frac{e^{-\frac{3}{2} W\left(-\beta \tilde{k}^{2} / a^{2}\right)}}{1+W\left(-\beta \tilde{k}^{2} / a^{2}\right)} .
\end{aligned}
$$

Here, $W$ is the Lambert $W$-function, defined via $W(x) e^{W(x)}=x[25]$ and $\tilde{k}^{i}=a \rho^{i} e^{-\beta \rho^{2} / 2}$ where $\rho^{i}$ is the Fourier transform of the physical coordinate $x^{i}$. $\tilde{k}^{i}$ is a variable that is equivalent to comoving momentum at large wavelengths.

Historically, the gauge invariant parameter $u$ was introduced to rewrite $S_{S}^{(1)}$ in the form of an action for a scalar field with a time-dependent mass $z^{\prime \prime} / z$ in Minkowskian space-time $[26]$ :

$$
S_{S}^{(2)}=\frac{1}{2} \int d \tau d^{3} \mathbf{y}\left(\left(\partial_{\tau} u\right)^{2}-\delta^{i j} \partial_{i} u \partial_{j} u+\frac{z^{\prime \prime}}{z} u^{2}\right)
$$

The resemblance of $S_{S}^{(2)}$ with the action of a massive scalar field in a Minkowskian background simplifies the task of choosing the initial conditions. Hence, the vacuum can be chosen following a similar procedure to that in Minkowskian space-time. The equation of motion derived from the cutoff modified $S_{S}^{(2)}$ is:

$$
u_{\tilde{k}}^{\prime \prime}+\frac{\kappa^{\prime}}{\kappa} u_{\tilde{k}}^{\prime}+\left(\mu-\frac{z^{\prime \prime}}{z}\right) u_{\tilde{k}}=0 .
$$

The difference between $S_{S}^{(1)}$ and $S_{S}^{(2)}$ is a boundary term

$$
\triangle S_{S} \equiv S_{S}^{(1)}-S_{S}^{(2)}=\int d \tau d^{3} \mathbf{y} \frac{d}{d \tau}\left(\frac{z^{\prime}}{z} u^{2}\right)
$$

that can be discarded as long as we have not implemented the cutoff.

However the minimal length hypothesis transforms the boundary term (15) in the following manner in $\tilde{k}$ space [19]

$$
\triangle S_{S} \rightarrow \int d \tau d^{3} \tilde{k} \kappa(\tau, \tilde{k}) \frac{d}{d \tau}\left(\frac{z^{\prime}}{z} u^{2}\right)
$$


or equivalently it adds

$$
-\int d \tau d^{3} \tilde{k} \frac{d \kappa(\tau, \tilde{k})}{d \tau}\left(\frac{z^{\prime}}{z} u^{2}\right)
$$

to the bulk term of the Lagrangian. The difference between eqs. (10) and (14) arises from the variation of the additional term (17).

Following [24], we define the scalar amplitude as

$$
A_{S}(k) \equiv \frac{2}{5} P_{S}^{1 / 2}=\frac{2}{5} \sqrt{\frac{k^{3}}{2 \pi^{2}}}\left|\frac{u_{\tilde{k}}}{z}\right|_{\tilde{k} / a H \rightarrow 0} .
$$

Perturbing the Einstein-Hilbert action about a homogenous, isotropic, spatially flat background as in (3), one can write down the action for tensor perturbations in the transverse traceless gauge as [24]

$$
S_{T}^{(1)}=\frac{m_{P l}^{2}}{64 \pi} \int d \tau d^{3} \mathbf{y} a^{2}(\tau) \partial_{\mu} h_{j}^{i} \partial^{\mu} h_{i}{ }^{j} .
$$

As in the scalar case, one can rewrite $S_{T}^{(1)}$ in the form of an action for a tensor field with time dependent mass in a Minkowski background by introducing the new variable $P^{i}{ }_{j}(y) \equiv$ $\sqrt{\frac{m_{P l}^{2}}{32 \pi}} a(\tau) h^{i}{ }_{j}(y)$

$$
S_{T}^{(2)}=\frac{1}{2} \int d \tau d^{3} \mathbf{y}\left(\partial_{\tau} P_{i}^{j} \partial^{\tau} P^{i}{ }_{j}-\delta^{r s} \partial_{r} P_{i}^{j} \partial_{s} P_{j}^{i}+\frac{a^{\prime \prime}}{a} P_{i}{ }^{j} P_{j}^{i}\right) .
$$

where $S_{T}^{(2)}$ differs from $S_{T}^{(1)}$ by a boundary term

$$
\triangle S_{T} \equiv S_{T}^{(2)}-S_{T}^{(1)}=\frac{32 \pi}{m_{P l}^{2}} \int d \tau d^{3} \mathbf{y}\left(\alpha P_{i}^{j} P^{i}{ }^{\prime}\right)^{\prime} .
$$

Incorporating the minimal length hypothesis (1) into $S_{T}^{(1)}$ and $S_{T}^{(2)}$ respectively yields the following equations of motion for the $\tilde{k}$-Fourier transform of $P_{i j}$ [19]

$$
\begin{gathered}
p_{\tilde{k}}^{\prime \prime}+\frac{\kappa^{\prime}}{\kappa} p_{\tilde{k}}^{\prime}+\left(\mu-\frac{a^{\prime \prime}}{a}-\frac{a^{\prime}}{a} \frac{\kappa^{\prime}}{\kappa}\right) p_{\tilde{k}}=0, \\
p_{\tilde{k}}^{\prime \prime}+\frac{\kappa^{\prime}}{\kappa} p_{\tilde{k}}^{\prime}+\left(\mu-\frac{a^{\prime \prime}}{a}\right) p_{\tilde{k}}=0 .
\end{gathered}
$$

These equations of motion differ because the minimal length hypothesis implies

$$
\triangle S_{T} \rightarrow \int d \tau d^{3} \tilde{k} \kappa(\tau, \tilde{k}) \frac{d}{d \tau}\left(\alpha P_{i}{ }^{j} P^{i}{ }_{j}\right)
$$

thereby modifying the boundary term eq.(21) in a nontrivial manner [19]. Following [24], we define the tensor amplitude as

$$
A_{T}(k) \equiv \frac{1}{10} P_{T}^{1 / 2}=\frac{1}{10} \sqrt{\frac{k^{3}}{2 \pi^{2}}}\left|h_{\tilde{k}}\right|_{\tilde{k} / a H \rightarrow 0} .
$$




\section{Tensor/Scalar Ratio and the Violation of the Con- sistency Relation}

One can expand the ratio of tensor/scalar fluctuations in terms of the slow roll parameters in the absence of a cut-off. To first order it is $[22,24]$

$$
r \equiv \frac{A_{T}^{2}}{A_{S}^{2}}=\epsilon
$$

where

$$
\epsilon \equiv \frac{3 \dot{\phi}_{0}^{2}}{2}\left(V\left(\phi_{0}\right)+\frac{1}{2} \dot{\phi}_{0}^{2}\right)^{-1}=\frac{m_{P l}^{2}}{4 \pi}\left(\frac{H_{\phi}}{H}\right)^{2}
$$

with the $\phi$ subscript and over-dot respectively denoting differentiation with respect to $\phi$

and the cosmic time, $t$, related to conformal time $\tau$ by $t=\int a d \tau$. Both tensor and scalar fluctuations contribute to the anisotropy of the CMBR. Hence, to extract the characteristics such as spectral indices for each type of fluctuation we need to know $r$ [27, 28].

Since scalar and tensor perturbations originate from a single inflaton potential they are not independent. A hierarchy of consistency conditions links them together [24]. It has been argued that such conditions - if empirically verified - would offer strong support for the idea of inflation. Observational difficulties will probably render only the first consistency condition useful. The first of these consistency relations relates $r$ to the tensor spectral index, $n_{T}$, defined as

$$
n_{T}(k) \equiv \frac{d \ln A_{T}^{2}(k)}{d \ln k}
$$

To first order in slow-roll parameters $n_{T}$ can expanded, yielding

$$
n_{T}=-2 \epsilon,
$$

and so the first-order consistency relation takes the following form

$$
r \equiv \frac{A_{T}^{2}}{A_{S}^{2}}=-\frac{n_{T}}{2}
$$

in the absence of a cut-off.

In presence of minimal length the relation (26) is modified

$$
\frac{A_{T}^{2}}{A_{S}^{2}}=\epsilon\left|\frac{p_{k}}{u_{k}}\right|_{k / a H \rightarrow 0}^{2}
$$


where $u_{k}$ and $p_{k}$ will satisfy different differential equations contingent upon the choice of action in the presence of a cutoff. Furthermore, eq.(29) no longer holds true ${ }^{1}$.

Hence one expects that Planck scale physics will modify the consistency relation. Our predictions of course depend on the choice of the action for tensor and scalar perturbations. As noted above, for both tensor and scalar spectra there are two physically motivated actions, yielding four cases of interest that we will separately analyze below.

\subsection{The Mode Equations in a Power Law Background}

Before presenting our numerical results for the power spectra, it will be instructive to consider the explicit form of the mode equations $(10,14,22,23)$. A power-law inflationary background is described by

$$
a(t)=t^{p}, \quad a(\tau)=\left(\frac{\tau}{\tau_{0}}\right)^{q}, \quad q=\frac{p}{1-p}
$$

where $t(\tau)$ is the cosmic (conformal) time and $p>1$. Assuming that at $t=1, a(t)=1$ then $\tau_{0}=1 /(p-1)$. We will track the evolution of the modes numerically from when they are created at the time $\tau_{\tilde{k}} \equiv \tau_{0}\left(e \beta \tilde{k}^{2}\right)^{1 / 2 q}$, until $\tau \rightarrow 0$ at which point we calculate the power spectrum. To this end, we define a new variable, $y$, so that $\tau=\tau_{\tilde{k}}(1-y)$. It will prove convenient to work with the rescaled quantity $k=\tilde{k} e^{p / 2} / \tilde{k}_{\text {crit }}$., where $\tilde{k}_{\text {crit }}$ corresponds to the mode that crosses the horizon just before the Hubble radius reaches the minimal length scale, $\sqrt{\beta} H=1$. Explicitly it is given by

$$
\tilde{k}_{\text {crit }}=e^{-1 / 2} p\left(\beta p^{2}\right)^{(p-1) / 2} \text {. }
$$

With these definitions, the mode equation (10) becomes

$$
\begin{aligned}
& \ddot{u}_{\tilde{k}}-\frac{q}{1-y} \frac{W(5+3 W)}{(1+W)^{2}} \dot{u}_{\tilde{k}}-\left(\frac{e q^{2} k^{2 / p} W}{(1-y)^{-2 q}(1+W)^{2}}\right. \\
&\left.+\frac{q(q-1)}{(1-y)^{2}}+\frac{q^{2}}{(1-y)^{2}} \frac{W(5+3 W)}{(1+W)^{2}}\right) u_{\tilde{k}}=0
\end{aligned}
$$

where an overdot now denotes a derivative with respect to $y$, and the argument of the Lambert $\mathrm{W}$ function is $-e^{-1}(1-y)^{-2 q}$. The other mode equations (14),(22) and (23) may be obtained by dropping the final term in the parentheses and replacing $u_{\tilde{k}}$ with $p_{\tilde{k}}$ as necessary. The definition of $k$ was chosen to remove the explicit dependence upon the minimal length, but we now see that there is an added benefit to the choice of these variables. For large $p$, to very good approximation $q$ is -1 . In fact, actually setting $q=-1$ changes the equations

\footnotetext{
${ }^{1}$ We are grateful to A. Kempf for bringing this to our attention
} 
very little. To a very good approximation then, all the important dependence upon $p$ occurs through the factor $k^{2 / p}$. When written using the $k$ variable the behavior of the modes is independent of $\beta$, but following all the factors through we find that the normalization of the power spectrum varies as $\beta^{-1 / 2}$.

We start tracking the mode numerically just after it is created by solving the mode equations approximately for small $y$. The approximate solution enables us to choose our vacuum, and fix the normalization of the mode function. There is a branch cut in the Lambert $\mathrm{W}$ function when its argument is $-e^{-1}$, but since we will start following the mode numerically from some small but positive $y$ we may treat it as a removable singularity when we determine the approximate solution, since $W \sim-1+2 \sqrt{-q y}+O(y)$ as $y \rightarrow 0^{+}$. In general the asymptotic solution is expressible in terms of Hankel functions. The explicit form is dependent on the exact mode equation since it is the terms having the factor $(1+W)^{-2}$ that dominate as $y \rightarrow 0$.

\section{$3.2\left(S_{T}^{(1)}, S_{S}^{(2)}\right)$}

Let us first assume that the actions for tensor and scalar fluctuations are $S_{T}^{(1)}$ and $S_{S}^{(2)}$, respectively. Since in this case $u_{\tilde{k}}$ and $p_{\tilde{k}}$ satisfy different equations, the tensor/scalar ratio differs from the standard quantum field theory prediction. Solving equation (34) near $y=0$ with the method of dominant balance [29] (previously employed in other studies [17, 18, 20]); we find

$$
p_{k}(y)=D_{+} G(k, y)\left(1+\xi_{1}(k, y)\right)\left(1+\xi_{2}(k, y)\right)+D_{-} G^{*}(k, y)\left(1+\xi_{1}^{*}(k, y)\right)\left(1+\xi_{2}^{*}(k, y)\right)
$$

where

$$
\begin{aligned}
& G(k, y)=y^{3 / 4} H_{-3 / 4}\left(2 \sqrt{A_{k} y}\right) \\
& \xi_{1}(k, y)=-\frac{e k^{2 / p}}{6}(-q y)^{3 / 2}(2-3 \log y) \\
& \xi_{2}(k, y)=\frac{q y^{2}}{48}\left(3 q\left(16-59 e k^{2 / p}\right)+4 i\left(2+e k^{2 / p}\right)\left(3 i+7 q^{2} \sqrt{2+e k^{2 / p}}\right)+42 q e k^{2 / p} \log y\right)
\end{aligned}
$$

and

$$
A_{k}=-\frac{q}{4}\left(2+e k^{2 / p}\right) .
$$

The quantities $D_{-}$and $D_{+}$are constrained by the Wronskian condition which implies:

$$
\left|D_{+}\right|^{2}-\left|D_{-}\right|^{2}=-\eta_{\tilde{k}} \pi \sqrt{-q} e^{-3 / 2} .
$$


In a near de-Sitter background if $D_{-} / D_{+}$goes to zero when $\beta \rightarrow 0$, then the standard QFT result can be recovered [20]. However if $D_{-} / D_{+}$is constant in this limit, one cannot recover the standard QFT result as $\beta \rightarrow 0$ [18]. We conjecture that the same type of reasoning is valid in a power-law background, and therefore we still have freedom in choosing the vacuum. We shall proceed with the choice $D_{-}=0$, which corresponds to a Bunch-Davies-like vacuum.

This analytic solution can then be used as an initial condition to numerically integrate the differential equation from a point in the vicinity of the singular point until $\tau \approx 0$. At this point, we extract the late time amplitude of $u_{\tilde{k}}$. Figure 1 illustrates our results for the tensor amplitude for $p=500$. This value of $p$ is consistent with recent observations indicating that, the scalar spectral index, $n_{S}$, which for a power-law background in the absence of minimal length happens to be $1-2 / p$, is greater than 0.95 [31]. We also assume that $\beta=100^{2}$, which corresponds to a minimal length 100 times larger than Planck scale. This is a reasonable assumption in the framework of scenarios of large extra dimensions [30]. We see that the standard tensor power spectrum is modulated by oscillations, corresponding to a slow decrease in $H$ as the universe expands. Increasing $p$ (though still working with the rescaled variable $k$ ) does not change the qualitative features of the power spectrum, it only results in a shift of the $\log k$ axis to the left. Since $k$ appears in the mode equation as $k^{2 / p}$ and $\tilde{k}_{c r i t}$ scales as $p^{p}$ this rescaling of the axis can have a significant effect on the spectrum when we compare power spectra for different $p$ with a common set of units for $\tilde{k}$. As $p$ increases, the wavelength of the oscillations increases [18]. Also, as $k$ increases the frequency of the oscillation increases, though the amplitude decreases. As expected, when $k \rightarrow \infty$, we recover the standard field theory result. The left graph in Figure 1 illustrates the power spectrum for the modes that have a larger amplitude than those seeding the structure formation in Hubble patch. In the right graph we plot a window of $k$ where the amplitudes are of the same order as the modes that are precursors to structure formation, $10^{-5} \leq P_{S}^{1 / 2} \leq 10^{-4}$ [18], or equivalently with $p=500,10^{-7} \leq A_{T} \leq 10^{-6}$.

On the left in Figure 2 we plot the tensor spectral index for the range of wavelengths that lie outside our horizon. The existence of minimal length yields running from a blue to a red spectrum on such scales. This happens despite the fact that $\epsilon$, the first slow roll parameter, does not have a local minimum. This is a counterexample to generic result of [32], which claimes that if the spectral index is to run from a blue to a red spectrum there must be a local minimum in the slope of the potential. On the right in Figure 2, we graph $n_{T}$ in the observable range of $k$. While we see the expected oscillations about the standard value, the large $k$ behavior is now more difficult to understand. The increasing frequency of oscillations 

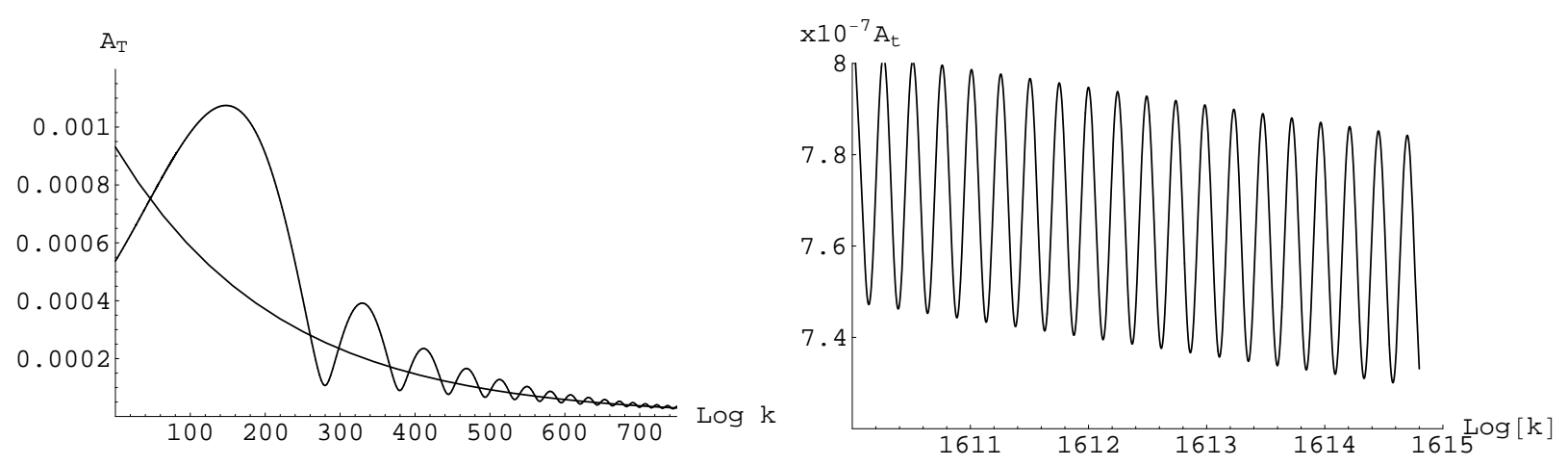

Figure 1: In these figures we assume that tensor perturbations are described by $S_{T}^{(1)}$. The left graph shows the dependence of $A_{T}$ on $\log k$ for $p=500$ and $\beta=10^{4}$. $k=1$ corresponds to $k_{\text {crit }}(500)$. The large modulation corresponds to physical scales much larger than our horizon. On the right, we plot the tensor amplitude with a window of $k$ whose amplitudes are of the same order as the modes that originated the structure formation in our universe.

for the tensor power spectrum results in a growth of both the amplitude and frequency of the oscillations. Current measurements put a lower bound of 40 on $p$ [31]. With such a weak lower bound, the frequency of the oscillations is very small. As any measurement of the spectral index is taken over a finite range of $k$, one would not be able to detect such oscillations. Taking small intervals centered at successively larger values of $k$ we would find that the average value of $n_{T}$ over the interval approaches the standard field theory result. To be able to detect these oscillations one needs extremely precise measurements. This oscillationary behavior of the spectral index is quite distinct from another model of transPlanckian physics based on the non-commutativity of physical time and space coordinates [33]. For such such a model, it was shown that the spectral index runs from $n>1$ on large scales to $n<1$, where transition happens on scales close to $H_{0}^{-1}[34,35,36,37]$.

Assuming that the action for scalar perturbations is described by $S_{S}^{(2)}$, the scalar modes satisfy eq.(23). Exploiting the dominant balance technique, we again extract the most singular terms in the mode equation in the vicinity of the irregular singular point with the approximate solution:

$$
u_{k}(y)=C_{+} F(k, y)\left(1+\epsilon_{1}(k, y)\right)\left(1+\epsilon_{2}(k, y)\right)+C_{-} F^{*}(k, y)\left(1+\epsilon_{1}^{*}(k, y)\right)\left(1+\epsilon_{2}^{*}(k, y)\right),
$$


where

$$
\begin{aligned}
& F(k, y)=y^{3 / 4} H_{-3 / 4}\left(2 \sqrt{B_{k} y}\right) \\
& \epsilon_{1}(k, y)=-\frac{e k^{2 / p}}{6}(-q y)^{3 / 2}(2-3 \log y) \\
& \epsilon_{2}(k, y)=-\frac{q y^{2}}{48}\left(48(1-q)+28 i q e^{3 / 2} k^{3 / p}+3 e k^{2 / p}(4+59 q-14 q \log y) .\right.
\end{aligned}
$$

and $B_{k}$ is given by

$$
B_{k}=-\frac{q}{4} e k^{2 / p}
$$

Again, we have a constraint on the integration constants $C_{+}$and $C_{-}$from the Wronskian condition:

$$
\left|C_{+}\right|^{2}-\left|C_{-}\right|^{2}=-\eta_{\tilde{k}} \pi \sqrt{-q} e^{-3 / 2}
$$

In the rest of the analysis, we choose $C_{-}=0$, to have a Bunch-Davies-like vacuum. However, we emphasize that this choice is not unique and there is still a considerable amount of freedom in the choice of $C_{-}$. Specifically, inspired by our analysis in near-de-Sitter space [20], we conjecture that if

$$
\lim _{\beta \rightarrow 0} \frac{C_{-}}{C_{+}}=0,
$$

we recover the standard result.

This approximate solution is again used to set the initial conditions for a numerical integration of the mode equation. The qualitative behavior of the scalar power spectrum is found to be similar to that found for the tensor modes. In Figure 3 we have plotted the tensor/scalar ratio for $p=500$ and $\beta^{1 / 2}=100$. The main effect of the different action for the scalar perturbations in this case appears to be a slight "compression" of the oscillations to smaller $k$. This compression causes the tensor/scalar ratio to oscillate in the observable window of $k$ about the constant value we expect to find when there is no minimum length. This is depicted on the right graph in Figure 3. Knowing this ratio is important if one is to understand the contribution that each of these types of perturbation makes to the anisotropy of the CMBR [27]. In Fig.3 we see from the left graph that the ratio stays constant at a value less than the standard QFT result for small values of $k$ that correspond to wavelengths outside our horizon. For increasing $k$ it attenuates until reaches a minimum, after which it increases to a value much higher than the standard result. Thereafter it starts oscillating about the standard QFT predictions. The amplitude of the oscillations dies off as $k$ increases. Notice also that $A_{T}^{2} / A_{S}^{2}$ is suppressed relative to $n_{T}$ by an order of magnitude, implying in general a violation of the consistency relation (30). 

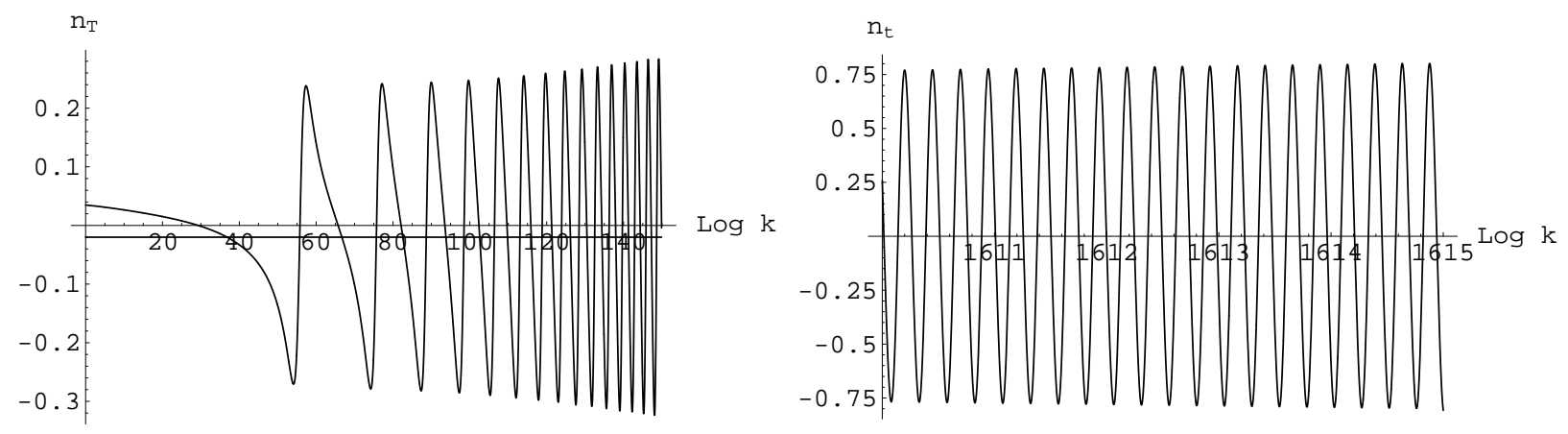

Figure 2: $S_{T}^{(1)}$ is assumed to be the action of tensor fluctuations. Left graph shows the dependence of $n_{T}$ on $\log k$ for wavelengths far bigger than our horizon. $\beta$ and $p$ are assumed to be $10^{4}$ and 500, respectively. The horizontal line represents the result when there is no minimum length. On the right we have graphed $n_{T}$ in the observable range of $k$.

This behavior for the tensor/scalar ratio was anticipated from our earlier calculations in near de-Sitter background [20], using $S_{T}^{(1)}$ for tensor and $S_{S}^{(2)}$ for scalar perturbations. In near de-Sitter space for small values of $\sigma \equiv \sqrt{\beta} H$, the ratio oscillates around the quantum field-theoretic prediction. For a fixed value of minimal length, this corresponds to small values of the Hubble parameter. As in a power-law background, short wavelength modes (large $k$ ) experience a slower rate of expansion, and so we expect oscillationary behavior in this region. Larger wavelengths are generated at the beginning of inflation, when the Hubble parameter (and in turn $\sigma$ ) were larger. For such wavelengths, this ratio is almost constant in a near de-Sitter background. In a power-law background for such values of $k$ we see (Fig.2) that the ratio is constant.

\section{3 $\left(S_{T}^{(2)}, S_{S}^{(1)}\right)$}

In this section, we assume that tensor and scalar perturbations satisfy eqs.(23) and (10) respectively. In a power-law background, $z^{\prime \prime} / z=a^{\prime \prime} / a$ [24] and $z^{\prime} / z=a^{\prime} / a$ [20] so the equation describing scalar (tensor) perturbations is the same as the one describing tensor (scalar) perturbations in section 4.1. From equation (31), one can deduce that $r / \epsilon$ now is just the inverse of $r / \epsilon$ from the last section.

In Figure 4 we show the tensor spectral index derived from the action $S_{T}^{(2)}$ overlaid on that found from $S_{T}^{(1)}$. Again, we note that the removal of the third term in parentheses of (34) causes a compression of the oscillations to smaller values of $k$, but the magnitude of oscillations is still larger than those of $A_{T}^{2} / A_{S}^{2}$ by an order of magnitude, indicating in 

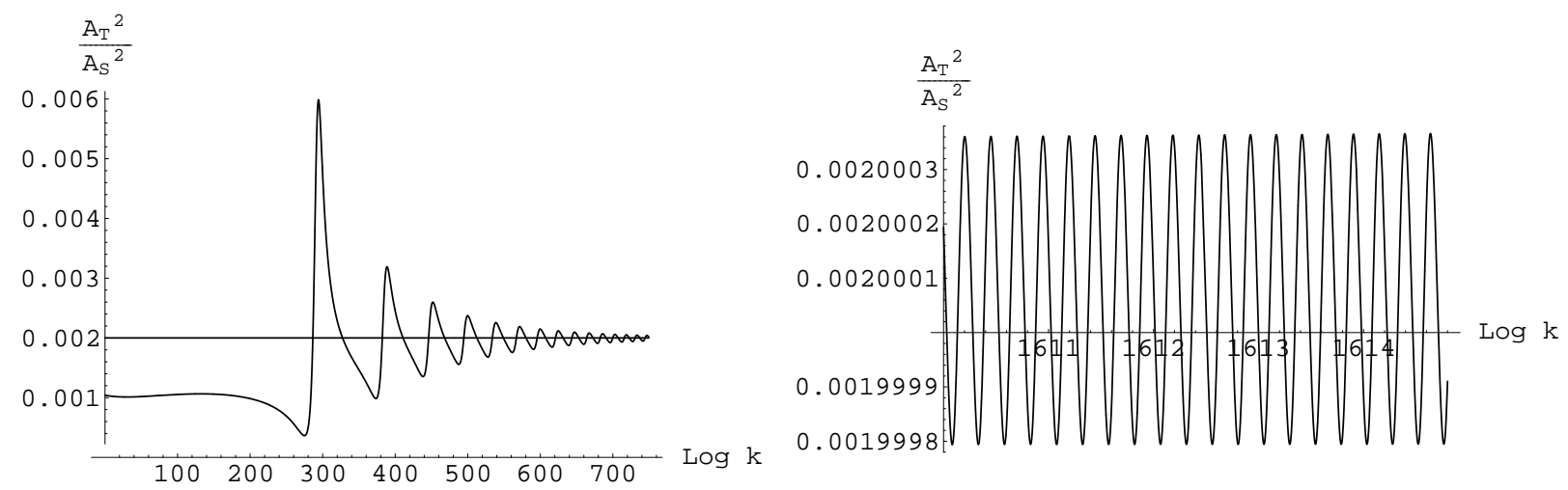

Figure 3: We assume $\left(S_{T}^{(1)}, S_{S}^{(2)}\right)$ respectively describe tensor and scalar fluctuations. The left figure shows $r$ for $p=500$ and $\beta=100^{2}$ for wavelengths far bigger than our horizon. On the right we plot the ratio of tensor to scalar fluctuations, in the observable window of $k$.

general that the consistency condition is still violated.

\section{$3.4\left(S_{T}^{(1)}, S_{S}^{(1)}\right)$ and $\left(S_{T}^{(2)}, S_{S}^{(2)}\right)$}

For both of these cases the mode equations for tensor and scalar fluctuations are identical. We therefore recover the standard field theory result for the ratio $A_{T}^{2} / A_{S}^{2}$. The tensor spectral index has already been presented in Figures 1 and 3. Again the oscillations about the standard result indicate there are violations of the consistency condition.

\section{$3.5 \beta$ dependence of fluctuations}

Up until now we have been working with a rescaled variable that allows us to study the generic behavior for any $\beta$. Recall that the definition of our variable $k$ involves $\beta$ dependence of the form $k \sim \beta^{(p-1) / 2} \tilde{k}$ and there is an overall factor of $\beta^{-1 / 2}$ in the normalization of the power spectrum. One may then qualitatively compare our results for different values of $\beta$ by noting that, up to normalization, changing $\beta$ just corresponds to a shifting of the $\log k$ axis. For example, a value of $\beta=100^{2}$, causes the spectra of Figures 1, 2 and 3 to shift to the left relative to the $\beta=500^{2}$ results. For a given $\tilde{k}$ the net result is that the size of the fluctuations about the standard field theory result are suppressed.

To be more exact, we may parameterize the tensor power spectrum as $A_{T}^{2}=A_{T, \mathrm{qft}}^{2}(1+$ $\left.\delta A_{T}(\beta, k)\right)$, where $A_{T, \mathrm{qft}}$ is the standard quantum field theory result for the tensor power spectrum. In Figure 4 , for action $S_{T}^{(2)}$, we plot $\delta A_{T}(\beta, k)$ for $\beta=500^{2}$ and $\beta=100^{2}$ written in units where $k=1$ corresponds to $\tilde{k}=e^{p / 2} / \tilde{k}_{\text {crit }}$ for $\beta=100^{2}$. We find that the size of the oscillations and their wavelength both appear to vary as $\beta^{1 / 2}$, the only dimensionful 

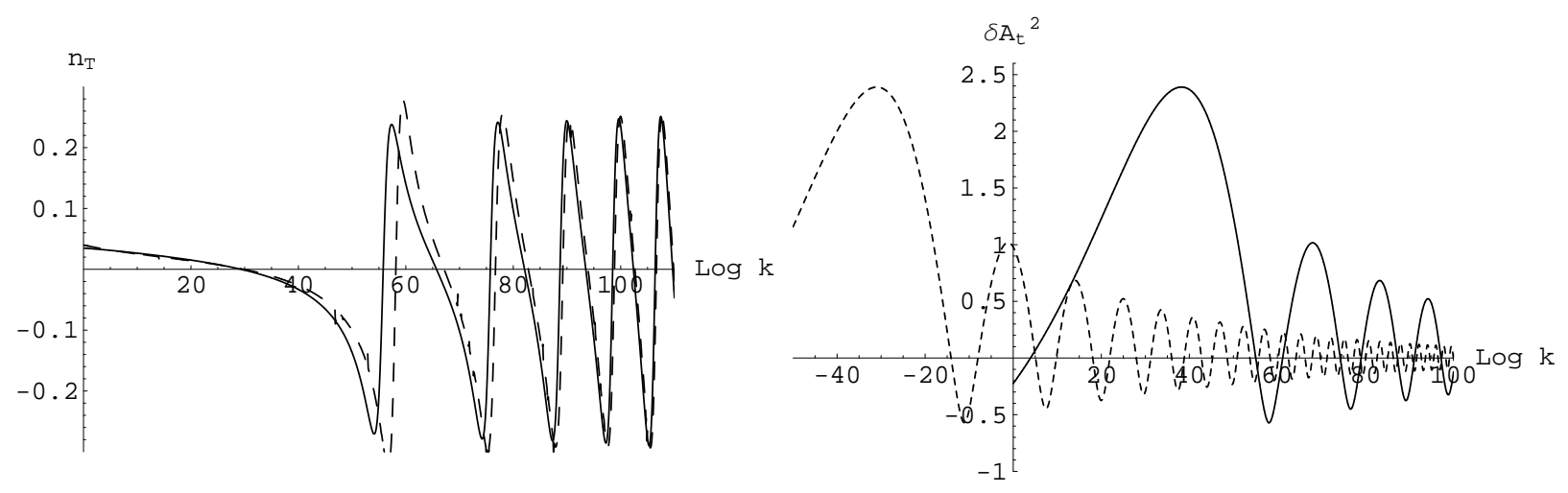

Figure 4: In the figure on the left, we overlay the tensor index for the power spectra obtained from $S_{T}^{(1)}$ (solid) and $S_{T}^{(2)}$ (dashed). On the right, we have graphed $\delta A_{T}$, modifications to the standard quantum field theory prediction due to the presence of minimal length, for $\beta=500^{2}$ (solid line) and $\beta=100^{2}$ (dashed). Here, we have assumed that $S_{T}^{(2)}$ describes the action for tensor perturbations and $p=100$.

parameter in the problem.

\section{Conclusion}

In this article we investigated the consistency relation between tensor and scalar fluctuations in the framework of power-law inflation with a cut-off due to minimal length. Since the method of implementing the minimal length hypothesis (1) depends on the action one starts from, there is a choice amongst an infinite number of actions that in the absence of minimal length are otherwise equivalent. However there are only two physically reasonable cases for both scalar and tensor perturbations: that of minimality (add no boundary terms to the original action) and that of simplicity (add terms such that the modified action most closely resembles the action of a free massive scalar field in a Minkowski background). This yields four distinct cases and we investigated each for a choice background consistent with recent observations that constrain the magnitude of the scalar spectral index.

Confining our attention to these cases, we found that Planck scale physics can considerably modify the consistency condition (30) and can lead to the running of spectral indices regardless of which action one employs. Depending on the choice of action for tensor and scalar perturbations, we may find that the tensor/scalar ratio oscillates (in the observable window of $k$ ) about the constant value we expect to find in the absence of minimal length. However the magnitude of the modifications depends upon the choice of action. Constraining this choice - both observationally and theoretically - remains a challenge for future studies. 


\section{Acknowledgement}

We are thankful to A. Kempf, R. Easther and W. H. Kinney for helpful discussions. This work was supported by the Natural Sciences \& Engineering Research Council of Canada.

\section{References}

[1] Robert H. Brandenberger, hep-ph/9910410.

[2] A. D. Linde, Particle Physics and Inflationary Cosmology (Harwood Academic, Chur, Switzerland).

[3] J. Martin \& R. H. Brandenberger, Phys. Rev. D63, 123501 (2001), hep-th/0005209; R. H. Brandenberger \& J. Martin, Mod. Phys. Lett. A16, 999 (2001), astro-ph/0005432; J. Martin, R. H. Brandenberger, Phys. Rev. D65, 103514

[4] B. Greene, K. Schalm, G. Shiu, J. P. van der Schaar, astro-ph/0503458

[5] W. Unruh, Phys. Rev. D51, 2827 (1995)

[6] S. Corely \& T. Jacobson, Phys. Rev. D54, 1568 (1996), hep-th/9601073; S. Corely, Phys. Rev D57, 6280 (1998), hep-th/9710075

[7] D. J. H. Chung, E. W. Kolb \& A. Riotto, Phys. Rev. D65, 083516 (2002) hep$\mathrm{ph} / 0008126$

[8] G. E. Volovik, Phys. Rept. 351, 195 (2001), gr-qc/0005091; G. E. Volovik, Pisma Zh. Eksp. Teor. Fiz. 73, 182 (2001) [JETP Lett. 73, 162 (2001)], hep-th/0101286

[9] R. H. Brandenberger \& J. Martin, Int. J. Mod. Phys. A17, 3663 (2002), hep-th/0202142

[10] A. A. Starobinsky, Pisma Zh. Eksp. Teor. Fiz. 73, 415 (2001) [JETP Lett. 73, 371 (2001)], astro-ph/0104043

[11] T. Tanaka, astro-ph/0012431

[12] U. H. Danielsson, Phys. Rev. D66, 023511 (2002)

[13] G. L. Alberghi, R. Casadio, A. Tronconi, Phys. Lett. B579, 1 (2004), gr-qc/0303035; R. Easther, B. R. Greene, W. H. Kinney and G. Shiu, Phys. Rev. D66, 023518 (2002), hep-th/0204129 
[14] A. Kempf, Phys. Rev. D63, 083514 (2001), astro-ph/0009209

[15] A. Kempf, hep-th/9810215

[16] D. J. Gross and P. F. Mende, Nucl. Phys. B303, 407 (1988). ; D. Amati, M. Ciafaloni, G. Veneziano, Phys. Lett. B216 41 (1989)

[17] R. Easther, B. R. Greene, W. H. Kinney \& G. Shiu, Phys. Rev. D64, 103502 (2001), hep-th/0104102

[18] R. Easther, B. R. Greene, W. H. Kinney \& G. Shiu, Phys. Rev. D67 063508 (2003)

[19] A. Ashoorioon, A. Kempf, R. B. Mann, Phys. Rev. D71, 023503 (2005), astroph/0410139

[20] A. Ashoorioon, R. B. Mann, Nucl. Phys. B716, 261 (2005), gr-qc/0411056

[21] Amjad Ashoorioon, Robert B. Mann, Phys. Rev. D71, 103509 (2005), gr-qc/0410053

[22] A. A. Starobinsky, Sov. Astr. Lett., 11, 133 (1985); E. D. Stewart \& D. H. Lyth, Phys. Lett. B302, 171 (1993)

[23] L. Hui \& W. H. Kinney, Phys. Rev. D65 103507 (2002), astro-ph/0109107

[24] J. E. Lidsey, A. R. Liddle, E. W. Kolb, E. Copeland, T. Barreiro \& M. Abney, Rev. Mod. Phys. 69, 373, (1997)

[25] R. Corless, G. Gonnet, D. Hare, D. Jeffrey, and D. Knuth, Adv. Comput. Math. 5, 329 (1996).

[26] Sov. Phys. JETP 67, 1297 (1988) [Zh. Eksp. Teor. Fiz. 94N7, 1 (1988)]; V. F. Mukhanov, H. A. Feldman, R. H. Brandenberger, Phys. Rep. 215, 203 (1992)

[27] D. S. Salopek, Phys. Rev. Lett. 693602 (1992).

[28] A. R. Liddle, D. H. Lyth, Phys. Lett. B291 391 (1992).

[29] Carl M. Bender and Steven A. Orszag, "Advanced Mathematical Methods for Scientists and Engineers", McGrawHill, Inc., 1978.

[30] N. Arkani-Hamed, S. Dimopoulos \& G. Dvali, Phys. Lett. B429 263 (1998); I. Antoniadis, N. Arkani-Hamed, S. Dimopoulos \& G. Dvali, ibid 436, 257 (1998). 
[31] D. N. Spergel, et. al., Astrophys. J. Suppl. 148 (2003) 175, astro-ph/0302209

[32] D. J. H. Chung, G. Shiu \& M. Trodden, Phys. Rev. D68 063501, 2003, astro-ph/0305193

[33] R. Brandenberger and P. M. Ho, Phys. Rev. D 66, 023517 (2002), hep-th/0203119

[34] Q. G. Huang and M. Li, JHEP 0306, 014 (2003), hep-th/0304203

[35] Q. G. Huang and M. Li, JCAP 0311, 001 (2003), astro-ph/0308458

[36] Q. G. Huang and M. Li, Nucl. Phys. B713, 219 (2005) astro-ph/0311378

[37] S. Tsujikawa, R. Maartens and R. Brandenberger, Phys. Lett. B574, 141 (2003), astro$\mathrm{ph} / 0308169$ 